Rev. Inst. Flor. v. 27 n. 2 p. $177-181$ dez. 2015

http://dx.doi.org/10.4322/rif.2015.014

ISSN impresso 0103-2674/on-line 2178-5031

\title{
UTILIZAÇÃO DO SPAD-502 PARA A PREDIÇÃO DOS TEORES DE NITROGÊNIO EM MUDAS DE Croton urucurana Baill. (NOTA CIENTÍFICA) ${ }^{1}$
}

\author{
USE OF SPAD-502 TO PREDICT NITROGEN CONTENT \\ IN Croton urucurana Baill. SEEDLINGS (SCIENTIFIC NOTE)
}

\author{
Glaucia UESUGI ${ }^{2,7}$; João Ricardo FAVAN ${ }^{3}$; Cristiano Bueno de MORAES 4 \\ Thayana Cristina Rebello WANGINIAK ${ }^{5}$; Magali Ribeiro da SILVA ${ }^{6}$
}

\begin{abstract}
RESUMO - O clorofilômetro portátil SPAD-502 permite avaliar o aspecto nutricional da planta de forma rápida e não destrutiva, sendo utilizado na área agronômica, mas com poucas pesquisas na silvicultura. Dessa maneira, este trabalho teve por objetivo avaliar a viabilidade do uso do SPAD na predição dos teores de nitrogênio em mudas de Croton urucurana produzidas em diferentes doses de solução nutritiva. As leituras com o equipamento foram realizadas em quatro folhas do terço médio de cada muda, para a obtenção de uma média/muda. O delineamento experimental utilizado foi em blocos inteiramente casualizados, três tratamentos de doses crescentes de solução nutritiva, quatro parcelas, 12 plantas/parcela. Os resultados foram submetidos à análise de variância e teste Tukey ao nível de $5 \%$ de probabilidade. Houve correlação entre a leitura SPAD e o teor de $\mathrm{N}\left(\mathrm{R}^{2}=0,87\right)$, sendo que nas doses menos concentradas houve correlação positiva. O uso do SPAD pode ser indicado como uma alternativa para a predição do teor de nitrogênio em mudas da espécie Croton urucurana.
\end{abstract}

Palavras-chave: sangra-d'água; clorofilômetro; nitrogênio; produção de mudas.

\begin{abstract}
SPAD-502 portable chlorophyll meter allows to evaluate the nutritional aspect of plant quickly and non-destructively, being widely used in agronomy, but there are few researches in silviculture. Thus, this study aimed to assess the use of SPAD device in predicting the nitrogen content of Croton urucurana seedlings in different doses of nutritive solution. SPAD values were performed in four leaves located in the middle third of seedling and in order to obtain average/plant. The experiment had a randomized block design with three treatments of increasing doses of nutritive solution, four plots of 12 plants in each treatment. The results were submitted to analysis of variance and Tukey test at $5 \%$ probability. There was correlation between SPAD readings and $\mathrm{N}$ content $\left(\mathrm{R}^{2}=0.87\right)$, and there was a positive correlation in less concentrated doses. The use of the SPAD-502 may be indicated as an alternative to predict the nitrogen content of Croton urucurana seedlings.
\end{abstract}

Keywords: sangra-d'água; chlorophyll meter; nitrogen; seedlings production.

\footnotetext{
${ }^{1}$ Recebido para análise em 07.01.15. Aceito para publicação em 22.09.15.

${ }^{2}$ Aluna de Doutorado em Ciência Florestal, Faculdade de Ciências Agronômicas de Botucatu - FCA/UNESP, Fazenda Experimental Lageado, Rua José Barbosa de Barros, 1780, 18610-307 Botucatu, SP, Brasil.

${ }^{3}$ Aluno de Mestrado em Ciência Florestal, Faculdade de Ciências Agronômicas de Botucatu - FCA/UNESP, Fazenda Experimental Lageado, Rua José Barbosa de Barros, 1780, 18610-307 Botucatu, SP, Brasil.

${ }^{4}$ Universidade Federal do Tocantins - Unidade de Gurupi, Rua Padejós, L 7 Chácara 69/72 Zona Rural, 77402-970 Gurupi, TO, Brasil.

${ }^{5}$ Aluna de Mestrado em Ciência Florestal, Faculdade de Ciências Agronômicas de Botucatu - FCA/UNESP, Fazenda Experimental Lageado, Rua José Barbosa de Barros, 1780, 18610-307 Botucatu, SP, Brasil.

${ }^{6}$ Faculdade de Ciências Agronômicas de Botucatu - FCA/UNESP, Departamento de Ciência Florestal, Fazenda Experimental Lageado,

Rua José Barbosa de Barros, 1780, 18610-307 Botucatu, SP, Brasil.

${ }^{7}$ Autor para correspondência: Glaucia Uesugi - ci_uesugi@yahoo.com.br
} 


\section{INTRODUÇÃO}

O conhecimento sobre as necessidades nutricionais de espécies florestais brasileiras ainda é muito incipiente (Sorreano et al., 2012), sendo importante estudos nesta área para estabelecimento de povoamentos florestais de boa qualidade e quantidade.

A metodologia padrão utilizada para análise química de acúmulo de nutrientes na planta é cara e requer tempo e a destruição dos tecidos foliares. $\mathrm{O}$ uso de clorofilômetros portáteis pode ser uma alternativa para se avaliar o estado nutricional das plantas, pois fornece resultados de forma rápida, não destrutiva e a custo baixo (Jesus e Marenco, 2008).

O Soil Plant Analysis Development (SPAD-502) é um tipo de clorofilômetro que apresenta medições dos teores indiretos de clorofila por meio de princípios ópticos baseados na absorbância e/ou refletância da luz pelas folhas, fornecendo uma leitura em unidades arbitrárias 0 a 99,9 SPAD (Minolta, 1989), correspondendo a um índice de cor verde. Considerando que 50 a $70 \%$ do $\mathrm{N}$ total na folha está associado a enzimas presentes nos cloroplastos (Chapman e Barreto, 1997), suas medições têm sido correlacionadas com o teor de nitrogênio das folhas (Murdock et al., 2004).

Vários trabalhos têm demonstrado sua eficiência em diagnosticar o estado nutricional, como em mangueiras (Chang e Chang, 1998), citros (Li et al., 1998; Araújo et al., 2004), cereais (Argenta et al., 2001), milho (Rorie et al., 2011), eucaliptos (Moraes et al., 2012) e cacaueiro (Dantas et al., 2012). Entretanto, há outros trabalhos que não apontaram boa correlação, como em Eucalyptus globulus e Eucalyptus nitens (Pinkard et al., 2006) e cedro (Freiberger et al., 2013). Segundo Berg e Perkins (2004), as relações matemáticas entre o índice SPAD e o teor de nitrogênio variam entre as espécies, as condições ambientais e o estágio de desenvolvimento da planta de uma mesma espécie.

Dessa maneira, o objetivo do estudo foi avaliar a viabilidade do clorofilômetro portátil SPAD-502 na predição dos teores de nitrogênio em mudas da espécie Croton urucurana Baill. produzidas em diferentes doses de solução nutritiva.

\section{MATERIAL E MÉTODOS}

O experimento foi conduzido nas dependências do viveiro do Departamento de Ciência Florestal da Faculdade de Ciências Agronômicas - FCA/UNESP/Campus de Botucatu/SP. O clima predominante no local é o Cfa, caracterizado como clima temperado quente (mesotérmico) úmido, sendo a precipitação pluviométrica anual média de $1.508,8 \mathrm{~mm}$ e a temperatura média do mês mais quente é superior a $22^{\circ} \mathrm{C}$ (Cunha e Martins, 2009).

A espécie utilizada foi Croton urucurana Baill., pertencente à família Euphorbiaceae, popularmente conhecida como sangra-d'água. É uma espécie pioneira de rápido crescimento, que ocorre naturalmente da Bahia ao Rio Grande do Sul, atrativa à fauna da região, o que auxilia a dispersão dessa espécie, contribuindo para a restauração e recuperação florestal de áreas degradadas, principalmente matas ciliares (Lorenzi, 2002).

$\mathrm{O}$ delineamento experimental utilizado para a instalação do teste foi em blocos inteiramente casualizados, sendo três tratamentos com doses crescentes de solução nutritiva, quatro repetições, doze mudas/repetição.

As sementes foram colhidas no Parque Municipal Joaquim Amaral Amando de Barros, localizado em área urbana no município de Botucatu-SP, nos meses de fevereiro e março de 2012. Tão $\operatorname{logo}$ ocorreu a colheita, a semeadura foi feita em substrato comercial denominado Carolina Soil®, constituído por turfa, casca de arroz carbonizada e vermiculita. As mudas foram produzidas em tubetes de polipropileno de $120 \mathrm{~cm}^{3}$ e a água utilizada para irrigação tinha $\mathrm{pH}$ de 6,0 e condutividade elétrica (CE) de $0,0057 \mathrm{dS} \mathrm{m}^{-1}$.

Durante o ciclo de produção, as mudas foram fertirrigadas com doses crescentes de solução nutritiva (três tratamentos), a fim de obter mudas com diferentes padrões nutricionais, ou seja, com distintos valores de índice de cor verde (SPAD). As soluções nutritivas foram constituídas das seguintes substâncias: nitrato de cálcio, ureia, monoamoniofosfato (MAP) purificado, cloreto de potássio e sulfato de magnésio. Tambémfoi acrescentado $1 \mathrm{~mL} \mathrm{~L}^{-1}$ de solução de micronutrientes constituída por ácido bórico, sulfato de manganês, sulfato de zinco, sulfato de cobre, molibdato de sódio e ferro. As condutividades elétricas das soluções nutritivas foram 1,$91 ; 2,18$ e 2,90 dS m $\mathrm{m}^{-1}$. 
Aos 188 DAS (dias após a semeadura), quando as mudas apresentavam em média $26 \mathrm{~cm}$ de altura e 4,56 $\mathrm{mm}$ de diâmetro do colo, e foram consideradas aptas para a expedição para o campo, foi determinado o teor indireto de clorofila por meio do aparelho medidor portátil Chlorophyll Meter, Modelo SPAD-502 (Minolta, 1989), sendo que as leituras foram realizadas nas folhas do terço médio, em quatro folhas por muda, evitando-se a nervura central, para a obtenção de uma média por planta.

As partes aéreas das mudas foram cortadas e levadas para secagem em estufa a $65{ }^{\circ} \mathrm{C}$ até obtenção de massa constante. Depois, a massa seca das partes aéreas foi medida, triturada e encaminhada ao Laboratório de Nutrição Mineral de Plantas do Departamento de Solos e Recursos Ambientais da Faculdade de Ciências Agronômicas da UNESP para determinação do teor de nitrogênio, conforme metodologia de Malavolta et al. (1997).

A associação entre os dados de teor de nitrogênio e índice de cor verde (SPAD) foi realizada efetuando-se a análise do coeficiente de correlação de Pearson e, posteriormente, análise de regressão. Os dados dos teores e conteúdos de nitrogênio na planta foram submetidos à análise de variância e nos casos em que houve significância realizou-se o teste Tukey a $5 \%$ de probabilidade.

\section{RESULTADOS E DISCUSSÃO}

O coeficiente de correlação de Pearson entre o teor de nitrogênio foliar e a leitura do SPAD foi de 0,83 , demonstrando que, em mais de $80 \%$ dos casos, quando houve o aumento do teor de nitrogênio houve também um aumento no valor do SPAD ( $p$-valor $=0,006141)$. Essa correlação pode ser considerada alta, comparando-se com o observado por Hurtado et al. (2010) em milho produzido em condições controladas, que foi de $60 \%$, e Dantas et al. (2012) em cacaueiros. que foi de $74 \%$. Essa correlação pode ser bem variável, dependendo do estádio fenológico da espécie, como o observado por Godoy et al. (2008) em cafeeiros fertirrigados, no qual o índice SPAD tendeu a reduzir entre o período de florescimento e a colheita de grãos.

$\mathrm{O}$ teor de nitrogênio e a leitura SPAD apresentaram resposta quadrática às doses de solução nutritiva aplicadas, havendo correlação positiva até o teor de $21 \mathrm{~g} \mathrm{~kg}^{-1}$, quando o índice SPAD atingiu o valor de 42,4 (Figura 1).

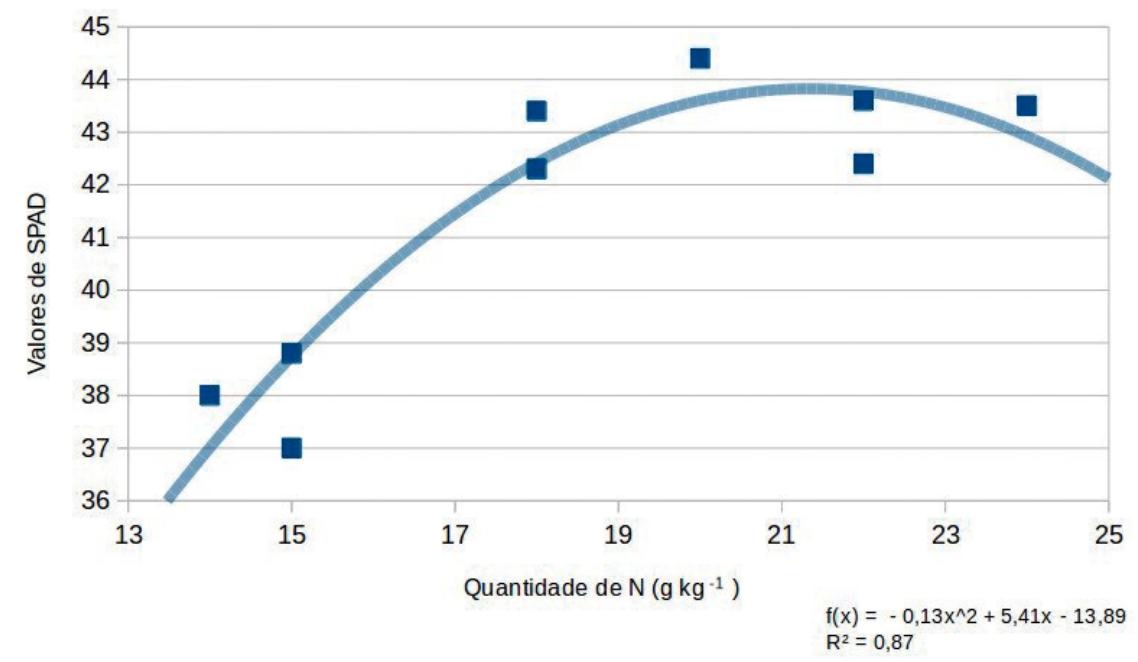

Figura 1. Correlação entre o teor de nitrogênio e o valor SPAD para mudas de sangra-d'água.

Figure 1. Correlation between nitrogen content and SPAD values for sangra-d'água seedlings. 
A solução nutritiva mais concentrada não implicou no aumento da intensidade da cor verde medida pelo clorofilômetro, o que sugere que houve um consumo de luxo da espécie, já que, nesta situação, o nitrogênio absorvido fica armazenado na forma de nitrato, não se associando à molécula de clorofila e, portanto, não sendo detectado pelo clorofilômetro (Dwyer et al., 1995).
Essa hipótese também foi corroborada ao analisar o teor e conteúdo de nitrogênio da parte aérea, pois nota-se que a aplicação de solução nutritiva mais concentrada não refletiu nos aumentos de teor e conteúdo de $\mathrm{N}$ na planta. Portanto, fica evidente que houve um desperdício de nutrientes nesse tratamento (Tabela 1).

Tabela 1. Médias do teor e conteúdo de nitrogênio em mudas de Croton urucurana aos 188 dias após a semeadura.

Table 1. Average of nitrogen content and concentration of Croton urucurana at 188 days after sowing.

\begin{tabular}{ccc}
\hline Solução nutritiva & Teor $\left(\mathrm{g} \mathrm{Kg}^{-1}\right)$ & Conteúdo (g/planta) \\
\hline padrão & $14,00 \mathrm{~b}$ & $37,52 \mathrm{~b}$ \\
$50 \%$ mais concentrada do que a padrão & $20,00 \mathrm{ab}$ & $61,00 \mathrm{a}$ \\
$100 \%$ mais concentrada do que a padrão & $21,00 \mathrm{a}$ & $61,11 \mathrm{a}$ \\
\hline
\end{tabular}

Médias seguidas pela mesma letra não diferem significativamente de acordo com o teste Tukey $(p<0,05)$.

Means followed by the same letter are not significantly different according to the Tukey test $(\mathrm{p}<0.05)$

Em mudas de Myracrodruon urundeuva e Luetzelburgia auriculata, Silva et al. (2012) observaram que o índice SPAD foi afetado de forma inversamente proporcional quando a salinidade ultrapassou $2,7 \mathrm{dS} \mathrm{m}^{-1}$. No presente estudo, encontrou-se resultado semelhante, obtendo-se uma correlação negativa entre teor de $\mathrm{N}$ e leitura de SPAD no tratamento da solução nutritiva cuja condutividade elétrica foi de $2,90 \mathrm{dS} \mathrm{m}^{-1}$. Segundo Parida e Das (2004), o teor de clorofila nas folhas tende a reduzir sob estresse salino.

Uma vez encontrada uma equação que explica a correlação em mais de $87 \%$ dos casos, o uso do clorofilômetro pode ser uma alternativa promissora para a avaliação do estado nutricional de mudas, pois se trata de um método não destrutivo (não prejudicando as mudas, que nesta fase apresentam poucas folhas devido ao seu pequeno porte), prático, instantâneo e de menor custo considerando a vida útil do aparelho e os custos de análises químicas.

\section{CONCLUSÕES}

A alta correlação existente entre 0 teor de nitrogênio, obtido analiticamente, e o índice de cor verde, dado pelo clorofilômetro SPAD, permite concluir que este equipamento tem sensibilidade para detectar variações no teor de nitrogênio de mudas de sangra-d'água em resposta a diferentes doses de solução nutritiva, com a vantagem de ser um método não destrutivo e de determinação rápida.

\section{AGRADECIMENTOS}

A autora Glaucia Uesugi agradece à Coordenação de Aperfeiçoamento de Pessoal de Nível Superior - CAPES pelo financiamento da bolsa de mestrado.

\section{REFERÊNCIAS BIBLIOGRÁFICAS}

ARAÚJO, R.A. et al. Características biométricas, índice SPAD-502 e emissão da fluorescência em porta-enxertos de citros. Revista Ceres, v. 51, n. 294, p. 189-199, 2004.

ARGENTA, G. et al. Clorofila na folha como indicador do nível de nitrogênio em cereais. Ciência Rural, v. 31, n. 4, p. 715-722, 2001.

BERG, A.K. van den; PERKINS, T.D. Evaluation of a portable chlorophyll meter to estimate chlorophyll and nitrogen contents in sugar mapple (Acer saccharum Marsh.) leaves. Forest Ecology and Management, v. 200, p. 113-117, 2004. 
CHANG, C.S.; CHANG, L.R. Two rapid determination methods for total chlorophyll content in fruit tree leaves. Bulletin of Taichung District Agricultural Improvement Station, v. 59, p. 37-45, 1998.

CHAPMAN, S.C.; BARRETO, H.J. Using a chlorophyll meter to estimate specific leaf nitrogen of tropical maize during vegetative growth. Agronomy Journal, v. 89, p. 557-562, 1997.

CUNHA, A.R.; MARTINS, D. Classificação climática para os municípios de Botucatu e São Manuel, SP. Revista Irriga, v. 14, n. 1, p. 1-11, 2009.

DANTAS, P.A.S. et al. Estimativa não destrutiva do teor foliar de nitrogênio em cacaueiro utilizando clorofilômetro. Revista Brasileira de Fruticultura, v. 34, n. 3, p. 669-677, 2012.

DWYER, L.M. et al. Quantifying the nonlinearity in chlorophyll meter response to corn leaf nitrogen concentration. Canadian Journal of Plant Science, v. 75, n. 1, p. 179-182, 1995.

FREIBERGER, M.B. et al. Crescimento inicial e nutrição de cedro (Cedrela fissilis Vell.) em função de doses de nitrogênio. Revista Árvore, v. 37, n. 3, p. 385-392, 2013.

GODOY, L.J.G. et al. Índice relativo de clorofila e o estado nutricional em nitrogênio durante o ciclo do cafeeiro fertirrigado. Revista Brasileira de Ciência do Solo, v. 32, p. 217-226, 2008.

HURTADO, S.M.C. et al. Sensibilidade do clorofilômetro para diagnóstico nutricional de nitrogênio no milho. Ciência e Agrotecnologia, v. 34, n. 3, p. 688-697, 2010.

JESUS, S.V.; MARENCO, R.A. O SPAD-502 como alternativa para determinação dos teores de clorofila em espécies frutíferas. Acta Amazonica, v. 38, n. 4, p. 815-818, 2008.

LI, Y.C. et al. A rapid nondestructive technique to predict leaf nitrogen status of grapefruit tree with various nitrogen fertilization practices. Hort Technology, v. 8, n. 1, p. 81-86, 1998.

LORENZI, H. Árvores brasileiras: manual de identificação e cultivo de plantas arbóreas nativas do Brasil. 4. ed. Nova Odessa: Instituto Plantarum, 2002. v. 1, 384 p.
MALAVOLTA, E.; VITTI, G.C.; OLIVEIRA, S.A. Avaliação do estado nutricional das plantas: princípios e aplicações. 2. ed. Piracicaba: POTAFOS, 1997. 319 p.

MINOLTA, C. Manual for chlorophyll meter SPAD-502. Osaka: Minolta Radiometric Instruments Divisions, 1989. 22 p.

MORAES, C.B. et al. Alterações morfológicas em Eucalyptus grandis sob aplicação de biorreguladores no período juvenil. Revista do Instituto Florestal, v. 24, n. 2, p. 251-257, 2012.

MURDOCK, L.; CALL, D.; JAMES, J. Comparision and use of chlorophyll meters on wheat (reflectance vs. transmittance/absorbance). Agricultural \& Natural Resources, v. 181, n. 11, p. 1-4, 2004. Disponível em: <http://http://www2.ca.uky.edu/agc/pubs/agr/ agr181/agr181.pdf $>$. Acesso em: 27 set. 2013.

PARIDA, A.K.; DAS, A.B. Salt tolerance and salinity effects on plants: a review. Ecotoxicology and Environmental Safety, v. 6, p. 324-349, 2004.

PINKARD, E.A.; PATEL, V.; MOHAMMED, C. Chlorophyll and nitrogen determination for plantation-grown Eucalyptus nitens and E. globulus using a non-destructive meter. Forest Ecology and Management, v. 223, p. 211-217, 2006.

RORIE, R.L. et al. Association of "greenness" in corn with yield and leaf nitrogen concentration. Agronomy Journal, v. 103, n. 2, p. 529-535, 2011.

SILVA, J.A. et al. Resposta fisiológica de Myracrodruon urundeuva e Luetzelburgia auriculata à salinidade. In: WORKSHOP INTERNACIONAL DE INOVAÇÕES TECNOLÓGICAS NA IRRIGAÇÃO, 4., 2012, Fortaleza. Resumos... Fortaleza, 2012. 5 p.

SORREANO, M.C.M; RODRIGUES, R.R.; BOARETTO, A.E. Guia de nutrição de espécies florestais nativas. São Paulo: Oficina de Textos, 2012. 254 p. 